\title{
A especialização inteligente na área central da cidade do Porto: domínios, ação pública e desafios
}

\author{
C. Ferreira (a), T. S. Marques (b), P. Guerra (c) \\ (a) CEGOT/Faculdade de Letras da Universidade do Porto, celiamarisaferreira@gmail.com \\ (b) CEGOT/Faculdade de Letras da Universidade do Porto, teresasamarques@gmail.com \\ (c) CEGOT / Faculdade de Letras da Universidade do Porto; IS -UP / Instituto de Sociologia / Universidade do Porto; Griffith Centre for Cultural \\ Research / Griffith University / Australia, mariadeguerra@gmail.com
}

\begin{abstract}
Resumen
A Comissão Europeia tem vindo a promover as estratégias de especialização inteligente como forma de cada país e, sobretudo, de cada região identificar áreas de investimento prioritárias, potenciando os seus principais ativos e tornando-os em vantagens competitivas face a outros territórios. A geografia da especialização inteligente regional reflete matizes resultantes da sua implementação à escala local. A estratégia de especialização inteligente da Região Norte de Portugal encontra-se patente nas abordagens de política pública do Município do Porto. Tendo como objeto de análise a área central da cidade do Porto, esta comunicação visa identificar e caracterizar os domínios de especialização inteligente mais evidenciados, bem como perceber as dinâmicas recentes e o papel da ação pública. Para o efeito, recorreu-se a uma abordagem predominantemente qualitativa, com recurso à análise de conteúdo de documentos estratégicos e à realização de entrevistas semi-diretivas a agentes locais dos diversos setores culturais, criativos e lúdicos.
\end{abstract}

Palavras chave: Baixa do Porto, Especialização inteligente, Política pública, Dinâmicas económicas.

\section{INTRODUÇÃO}

As estratégias de especialização inteligente foram promovidas pela Comissão Europeia no âmbito do processo de implementação da Estratégia Europa 2020. E, neste contexto, é seu objetivo potenciar as características e os ativos específicos de cada país e região, tornando-os em potencialidades competitivas face a outros territórios. A Estratégia Europa 2020 pressupõe a sua implementação a diferentes escalas, desde a europeia até à escala local, atribuindo particular importância à implementação regional. A necessidade de uma maior eficácia das políticas promotoras da inovação e competitividade e o reconhecimento de que one size does not fit all estão na origem da promoção de estratégias nas quais as regiões são consideradas parte fulcral ao nível da delineação, aplicação e gestão.

A CCDR-Norte foi responsável pela elaboração da estratégia regional de especialização inteligente da Região Norte de Portugal, identificando oito domínios estratégicos prioritários. A área central da cidade do Porto é caracterizada por uma elevada densidade de pessoas e atividades diversas e por uma forte concentração de equipamentos e instituições diversificados. Estes fatores concorrem para a criação de um ambiente propício à criatividade e à inovação, à fertilização cruzada de conhecimentos, ao estabelecimento de redes densas e de configuração variada entre os diversos agentes da área e, por conseguinte, a dinâmicas de competitividade territorial positivas e impulsionadoras de uma economia forte e sustentável. Neste contexto, dada a forte expressão de domínios de especialização inteligente definidos na estratégia regional e dado o facto da estratégia se encontrar patente nos instrumentos de atuação e nas medidas encetadas pelo Município do Porto, nos últimos anos, este trabalho visa identificar e caracterizar os domínios de especialização inteligente mais evidenciados na área central da cidade, bem como perceber as suas dinâmicas recentes e o papel da ação pública local. Para o efeito, recorreu-se a uma abordagem predominantemente qualitativa, com recurso à análise de conteúdo de documentos de estratégia pública local e à realização de oito entrevistas semi-diretivas, realizadas durante o ano de 2017, a agentes da ação pública e dos setores culturais, criativos e lúdicos locais. 


\section{A GÉNESE E AS ESTRATÉGIAS DE IMPLEMENTAÇÃO DA ESPECIALIZAÇÃO INTELIGENTE}

A abordagem da especialização inteligente, também designada por RIS3, teve origem nas estratégias de inovação regional, que tiveram um forte desenvolvimento a partir de 1994. O conceito surgiu no âmbito do desenvolvimento de trabalhos em torno da política de coesão da Comissão Europeia, realizados por Dominic Foray e pelo grupo de peritos Knowledge for Growth. O enfraquecimento dos sistemas nacionais de inovação como unidade de análise para a tomada de decisão e o fortalecimento, por sua vez, dos sistemas regionais e a emergência das redes globais de inovação propiciaram o enfoque nas estratégias de especialização inteligente. Este processo foi reforçado, mais recentemente, pelo reconhecimento por parte de muitos países de que estas estratégias constituem um instrumento mais eficaz para fazer face à crise económico-financeira, por via da liderança regional em matéria do desenvolvimento económico baseado no conhecimento intensivo e na inovação (OECD, 2013).

As estratégias nacionais e regionais de especialização inteligente foram promovidas pela Comissão Europeia no âmbito do processo de implementação da Estratégia Europa 2020. E, neste contexto, visam potenciar as características únicas e os ativos mais relevantes de cada país e região, tornando-os em potencialidades competitivas e em vantagens comparativas face a outros territórios (Ribeiro \& Ferrão, 2014).

A Estratégia Europa 2020 pressupõe a sua implementação a diferentes escalas, desde a europeia até à escala local, atribuindo particular ênfase à implementação regional (CE, 03/03/2010; ESPON, 2013; EU, 2012). A necessidade de uma maior eficácia ao nível da implementação das políticas de suporte à inovação e à competitividade e o reconhecimento de que one size does not fit all estão na origem da promoção, por parte da Comissão Europeia, das estratégias regionais de especialização inteligente nas quais as entidades regionais são parte fulcral na delineação, aplicação e gestão. Considerando-se que as regiões não conseguem ser economicamente muito competitivas em todos os setores, preconiza-se a realização de um exercício de avaliação e identificação das características e dos recursos sobre os quais focar a aplicação dos instrumentos de política pública para que estes tenham impactos significativos em matéria de competitividade, crescimento económico e emprego. A especialização inteligente traduz-se, ainda, na fertilização cruzada de atividades fortes na região, com o intento de gerar mais-valias capazes de atrair pessoas e investimento (CCDR-N, Sem data). No momento atual, pós-crise económico-financeira de 2008, a abordagem da especialização inteligente afigura-se promissora no aumento da eficiência dos investimentos europeus em investigação, inovação e empreendedorismo (Marques, Queiroz, \& Alves, 2014).

O modelo analítico dos sistemas de produção de conhecimento e inovação da hélice quádrupla, apresentado por Carayannis e Campbell em 2011, vai ao encontro das estratégias de RIS3. Este modelo acrescenta às três hélices do modelo inicial de Leydesdorff e Etzkowitz - instituições governativas, academia e setor empresarial a sociedade civil e os cidadãos, considerando que a cultura, os valores, os estilos de vida das sociedades e os meios de comunicação social têm um papel influenciador nada menosprezável (Carayannis \& Campbell, 2011; Ferreira, 2013). Neste modelo, o utilizador final (cidadão, consumidor, utente, empresa, etc.) é envolvido diretamente no processo de inovação (Carvalho, 2013; Winden \& Carvalho, 2015). A implementação de políticas de inovação, enquadradas nas estratégias de especialização inteligente, norteia-se pelo modelo da hélice quádrupla, envolvendo os denominados utilizadores avançados (empresas, instituições de ensino superior e de I\&D, entidades públicas de conceção e gestão de políticas de I\&D, utilizadores de inovação ou entidades representativas da procura e do consumo) num processo integrado e de liderança colaborativa onde cada agente tem um papel pró -ativo (CCDR-N, Sem data). As estratégias de especialização inteligente incentivam os fluxos de conhecimento e as interligações entre os diversos agentes de inovação, a governação multinível e a criação de massa criativa e capital social (Marques \& Santos, 2013; Ribeiro \& Ferrão, 2014).

Os argumentos a favor desta abordagem consistem fundamentalmente: na aplicação mais eficiente dos recursos públicos; na criação de sinergias entre mecanismos de suporte público da I\&D e inovação, promoção industrial e instituições de formação; na identificação dos domínios mais promissores para o empreendedorismo e o crescimento económico através de uma análise cuidada dos objetivos, competências e vantagens competitivas das cidades, regiões ou países; na criação de mecanismos que permitem o desenvolvimento estratégico baseado em interações multifacetadas entre agentes diversificados; no mapeamento de atores chave; e na criação de sistemas de monitorização e avaliação baseados na evidência para selecionar domínios de conhecimento e projetos inovadores (Nijkamp \& Kourtit, 2013). A abordagem à escala urbana encontra-se relacionada com a seleção de prioridades, baseadas nas potencialidades e vantagens comparativas, com o objetivo de canalizar meios e desenvolver estratégias para que a investigação e a inovação vão ao encontro das necessidades de mer- 
cado, alavancando oportunidades emergentes. As cidades detêm importantes concentrações de instituições e atividades de I\&D, o que as torna motores da competitividade às escalas regional e nacional (EU \& UN-Habitat, 2016; Santos \& Marques, 2013).

\section{ESPECIALIZAÇÃO INTELIGENTE NO NORTE DE PORTUGAL E NA ÁREA METROPOLITANA DO PORTO}

A nível da realidade portuguesa, a implementação regional das estratégias de especialização inteligente foi atribuída às Comissões de Coordenação e Desenvolvimento Regional (CCDR). A CCDR da Região Norte (CCDRN) foi responsável pela elaboração da estratégia regional de especialização inteligente da Região Norte de Portugal, na qual se encontram identificados oito domínios estratégicos (Figura 1) para o período de programação 2014 -2020 (CCDR-N, Sem data).

Alinhada com esse documento, bem como com a Estratégia Europa 2020, a estratégia de base territorial para o horizonte 2020 para a Área Metropolitana do Porto (AMP) preconiza o crescimento económico sustentável, através de respostas diferenciadas e adequadas aos desafios específicos da área. Para o efeito, os principais objetivos estabelecidos passam pela integração de instituições e atividades nas redes globais, procurando uma posição de liderança, e pelo apoio e facilitação da inovação. A especialização inteligente é considerada como um modo de atrair e reter talento e de potencializar recursos de produção de conhecimento e inovação (Marques et al., 2014).

\begin{tabular}{|c|c|}
\hline Domínios & Racional de especialização inteligente \\
\hline Ciências da Vida e Saúde & $\begin{array}{l}\text { Consolidação das dinâmicas de articulação entre a investigação regional } \\
\text { e as empresas nas indústrias e serviços na área da saúde. }\end{array}$ \\
\hline Cultura, Criação e Moda & $\begin{array}{l}\text { Exploração do potencial das indústrias criativas (sobretudo nas áreas de } \\
\text { design e arquitetura), de novos materiais e de tecnologias de produção } \\
\text { inovadoras. }\end{array}$ \\
\hline Recursos do Mar e Economia & $\begin{array}{l}\text { Estabelecimento de relações de articulação entre engenharias aplicadas, } \\
\text { recursos do mar e atividades económicas que os valorizem. }\end{array}$ \\
\hline Capital Humano e Serviços Especializados & $\begin{array}{l}\text { Promoção de competências acumuladas na área das Tecnologias de } \\
\text { Informação e Comunicação (TIC) (em particular, no desenvolvimento de } \\
\text { aplicações multimédia e na programação e engenharia de sistemas). }\end{array}$ \\
\hline Indústrias da Mobilidade e Ambiente & $\begin{array}{l}\text { Aproveitamento das competências científicas nas áreas das tecnologias } \\
\text { de produção e dos materiais para promover o upgrade das indústrias de } \\
\text { componentes de automóveis e moldes. }\end{array}$ \\
\hline Sistemas Avançados de Produção & $\begin{array}{l}\text { Desenvolvimento de fileiras associadas às Tecnologias de Largo Espec- } \\
\text { tro, nomeadamente os Sistemas de Produção Avançados, as Nanotecno- } \\
\text { logias Materiais e as Tecnologias de Informação e Comunicação e Eletró- } \\
\text { nica (TICE). }\end{array}$ \\
\hline Sistemas Agroambientais e Alimentação & $\begin{array}{l}\text { Potencialização dos produtos de elevado valor acrescentado e das com- } \\
\text { petências científicas, tecnológicas e empresariais para dar resposta a } \\
\text { segmentos de procura mais dinâmicos. }\end{array}$ \\
\hline Capital Simbólico, Tecnologias e Serviços do Turismo & $\begin{array}{l}\text { Valorização de recursos culturais e intensivos em território, aproveitando } \\
\text { as capacidades científicas e tecnológicas, nomeadamente nas áreas da } \\
\text { gestão, marketing e TIC, e a oferta turística relevante. }\end{array}$ \\
\hline
\end{tabular}

Figura 1 - Domínios de especialização inteligente da Região Norte de Portugal.Fonte: Adaptado de CCDR-N (Sem data), pp. 30 e 31.

\section{E ENTÃO À ESCALA LOCAL? O CASO DA ÁREA CENTRAL DO PORTO.}

A área central da cidade do Porto, como é próprio das áreas centrais, é caracterizada por uma elevada densidade e diversificação de pessoas e atividades e por uma forte concentração de equipamentos e instituições de índole diversa. Estes fatores concorrem para a criação de um ambiente propício à criatividade e à inovação, à fertilização cruzada de conhecimentos, ao estabelecimento de redes densas e de configuração variada entre os diversos agentes da área e, por conseguinte, a dinâmicas de competitividade territorial positivas e impulsionado- 
ras de uma economia forte e sustentável. Neste contexto, e dada a expressão de domínios de especialização inteligente definidos na estratégia regional e dado o facto da estratégia de especialização inteligente definida para a Região Norte de Portugal se encontrar patente nas estratégias e nos instrumentos de atuação do Município do Porto, em anos recentes, este trabalho pretende identificar e caracterizar os domínios de especialização inteligente mais evidenciados na área central da cidade, analisando as suas dinâmicas recentes e o papel da ação pública local na sua potencialização. Para o efeito, recorreu-se a uma abordagem predominantemente qualitativa, recorrendo-se à análise de conteúdo de documentos de estratégia pública local e à realização de oito entrevistas semi-diretivas a decisores públicos e a agentes dos setores culturais, criativos e lúdicos locais. As entrevistas foram realizadas durante o ano de 2017. De referir ainda que a observação direta dos fenómenos mostrou-se essencial para aferir afirmações e confrontar leituras.

\begin{tabular}{|l|l|}
\hline \multicolumn{1}{|c|}{ Domínios } & \multicolumn{1}{c|}{ Descrição } \\
\hline Capital Simbólico, Tecnologias e Serviços de Turismo & $\begin{array}{l}\text { Sobressaem as atividades recreativas e de lazer, que enquadram a valori- } \\
\text { zação dos recursos culturais, patrimoniais e naturais, bem como as ativi- } \\
\text { dades de apoio ao setor do turismo (alojamentos, agências de viagem e } \\
\text { operadores turísticos), o marketing, o comércio a retalho e a restauração. }\end{array}$ \\
\hline Capital Humano e Serviços Especializados & $\begin{array}{l}\text { Estão representados na área central pelos serviços às empresas, pelas } \\
\text { indústrias criativas e pelas atividades de I\&D e atividades relacionadas } \\
\text { com as TIC. }\end{array}$ \\
\hline Cultura, Criação e Moda & $\begin{array}{l}\text { Reflete-se nas indústrias criativas, assim como nas atividades relaciona- } \\
\text { das com o vestuário e o calçado ou nos serviços pessoais e às empresas. }\end{array}$ \\
\hline Recursos do Mar e Economia & $\begin{array}{l}\text { É representado pelas atividades recreativas e de lazer, com particular } \\
\text { destaque para o turismo náutico (na vertente da prática de desporto e na } \\
\text { vertente dos transportes turísticos que operam no Rio Douro), pelos } \\
\text { serviços às empresas e pelo comércio a retalho e restauração. }\end{array}$ \\
\hline
\end{tabular}

Figura 2 - Domínios de especialização inteligente predominantes na área central do Porto. Fonte: Elaboração própria.

\section{DOMÍNIOS DE ESPECIALIZAÇÃO INTELIGENTE, AÇÃO PÚBLICA E PRINCIPAIS DESAFIOS}

Todos os oito domínios prioritários de especialização identificados na estratégia definida para a Região Norte se encontram presentes na cidade do Porto. $\mathrm{Na}$ área central da cidade destacam-se quatro domínios: o Capital Simbólico, Tecnologias e Serviços de Turismo; o Capital Humano e Serviços Especializados; a Cultura, Criação e Moda; e os Recursos do Mar e Economia (Figura 2).

Como podemos verificar pela análise da Figura 2, há diversas atividades que cruzam mais do que um domínio e cujas competências específicas entram na equação da fertilização cruzada de informação, conhecimentos e ideias, gerando mais-valias ao nível da competitividade económica da área e, através dela, da cidade no seu todo.

Desde logo, a elevada oferta de comércio e restauração é, desde longa data, uma característica da área central do Porto. Na atualidade, tradição e modernidade coexistem na mesma rua ou, até, no mesmo estabelecimento ou evento de natureza económica. Os estabelecimentos mais antigos procuram modernizar-se através da meIhoria do serviço prestado, potenciando a sua mais-valia que é, ao fim ao cabo, a venda de produtos que simbolizam as tradições e a identidade local. Os estabelecimentos mais modernos recorrem a estratégias de hibridismo e cosmopolitização, apresentando ambiências apelativas e vendendo, muitas vezes, produtos tradicionais reinventados ou com características globalizadas, atraindo um público mais volátil e muitos turistas (Ferreira, Marques \& Guerra, 2016a).

O turismo assume-se como um dos principais setores económicos da área central do Porto na atualidade. Há uma conjugação de fatores que concorrem para a elevada atratividade da área e para a grande afluência de turistas ao longo de todo o ano. A proliferação do fenómeno low-cost (voos e alojamento), aliada à elevada concentração de estabelecimentos hoteleiros e de operadores de animação turística, à elevada procura do produto city-break a nível nacional e internacional, aos, ainda, relativamente baixos preços da cidade e à segurança (comparativamente com outras cidades europeias) são fatores de atração de pessoas (Ferreira, Marques \& Guerra, 2016b). 


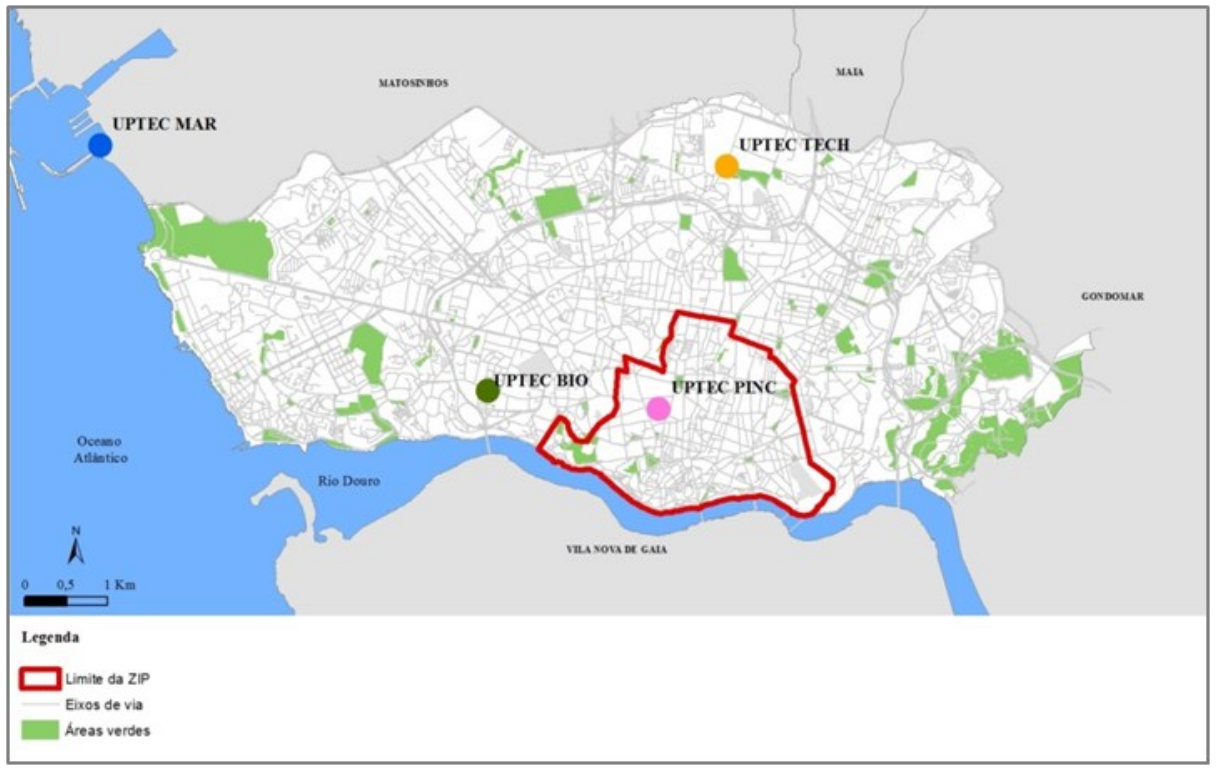

Figura 3 - Localização dos Polos da UPTEC ${ }^{1}$. Fonte: Elaboração própria.

Existem, no momento atual, elementos que concorrem para o reforço do capital sociocultural da área central do Porto. A diversidade de atividades, agentes e eventos que formam a economia cultural e criativa da área concorrem para a ascensão deste setor. Estamos a falar de atividades geridas por entidades de escalas de atuação diferenciadas e onde se encontram diferentes domínios de intervenção. Trata-se de atividades com capacidade para desempenhar um importante papel de adaptação das empresas aos avanços tecnológicos e a tendências de globalização dos mercados. No caso específico das indústrias criativas, de relevar a localização na área (Figura 3) do Polo das Indústrias Criativas do Parque de Ciência e Tecnologia da Universidade do Porto (UPTEC PINC).

O UPTEC PINC encontra-se em funcionamento desde janeiro de 2010 , constituindo um espaço de interação entre pessoas e projetos que procuram desenvolver e explorar a criatividade de forma empreendedora (em áreas como a arquitetura, as artes visuais, as artes performativas, o design, o audiovisual, a publicidade e a comunicação), sempre enquadradas no ambiente de I\&D da Universidade (UPTEC, Sem data). O UPTEC PINC constitui uma das estruturas basilares do Cluster de Indústrias Criativas da Região Norte de Portugal (Ribeiro \& Ferrão, 2014), sendo fortemente orientado para o mercado (Marques et al., 2017). De referir também a presença no local da incubadora Palácio das Artes - Fábrica de Talentos, gerida pela Fundação da Juventude, que existe desde 2009 para apoiar criadores individuais no desenvolvimento e promoção dos seus trabalhos.

A nível da atuação pública municipal, as políticas voltadas para a produção de conhecimento e inovação cimentam-se em redes de atores, formando uma hélice quádrupla que envolve empresas em diversos estados de maturação - desde start-ups a empresas bastante consolidadas no mercado -, instituições de ensino superior e de I\&D ligadas à Universidade do Porto, ao Instituto Politécnico do Porto e demais universidades privadas que se localizam na cidade, entidades públicas e organismos diversos que interagem em projetos diversos, que dinamizam e potenciam recursos humanos e tecnológicos. Pretende-se com esta abordagem a utilização eficiente dos limitados recursos públicos através da sua concentração em setores considerados capazes de alavancar processos de empreendedorismo, de crescimento económico e de reforço da competitividade da cidade no seu todo. Pretende-se captar investimento privado, criar dinâmicas de geração de emprego qualificado e criar condições favoráveis para o empreendedorismo. Nesta matéria, é pertinente referir dois projetos relevantes.

Um deles é o ScaleUp Porto, que tem uma projeção mais ampla, integrando a cidade do Porto no movimento ScaleUp For Europe. Baseia-se nos princípios da confiança, da facilitação de redes e parcerias, na aprendizagem conjunta e na partilha de conhecimento. Resulta de uma iniciativa do Município em parceria com o UPTEC e com

\footnotetext{
${ }^{1}$ ZIP é o acrónimo de Zona de Intervenção Prioritária. Trata-se de uma delimitação apresentada, em 2005, pela Sociedade de Reabilitação Urbana Porto Vivo (Porto Vivo, SRU) no âmbito do seu trabalho de requalificação e revitalização da Baixa portuense. Assume-se, para efeitos deste trabalho, como correspondendo à área central do Porto.
} 
o apoio da Agência Nacional de Inovação (Porto City Hall, 2016). Enquanto o UPTEC e outras incubadoras trabaIham na conceção e implementação de projetos de negócio e na criação de start-ups, o ScaleUp Porto procura trabalhar numa fase subsequente, em que as empresas procuram ganhar dimensão e são já capazes de gerar emprego qualificado. E, neste sentido, visa criar condições para que qualquer scale-up se instale e permaneça na cidade. São tendencialmente as empresas de base tecnológica que conseguem obedecer aos critérios exigidos, mas o projeto está, à partida, aberto a qualquer tipo de atividade. O Município procura através deste projeto reter capital humano, procurando assegurar níveis de qualidade de vida que atraiam e fixem pessoas altamente qualificadas na cidade. O outro projeto relevante é o Porto Innovation Hub. A área central acolheu a edição que decorreu em 2017. O principal objetivo é estabelecer uma ligação próxima entre o Município e o ecossistema de empresas e cidadãos (residentes ou visitantes). Envolve atividades como debates, rastreios de saúde, testes laboratoriais ou mostras de inovação. Pretende dar a conhecer empreendedores e os projetos por si desenvolvidos, projetando assim soluções tecnológicas inovadoras.

Perante o exposto anteriormente, impõe-se agora refletir sobre alguns desafios que se colocam à área central em matéria de domínios de especialização inteligente. Desde logo, coloca-se o desafio aos agentes e às atividades de manter a autenticidade e a identidade locais, ao mesmo tempo que se acompanha as tendências e exigências do mercado, reforçando os processos de competitividade. Um desafio específico se coloca ao setor do turismo: a turistificação excessiva da área (indiciada já pelo surgimento de lojas e produtos estereotipados) pode conduzir à perda de identidade, fator fulcral da elevada atração turística da área central do Porto. Por fim, identifica-se um terceiro desafio, mais voltado para a atuação e interação dos agentes. Desafio para que articulem interesses, estratégias e recursos, potenciando setores cuja capacidade e competências instaladas na área central do Porto os tornam bastante competitivos, inclusive a nível internacional. E desafio para que atuem sobre eventuais problemas e constrangimentos de forma concertada, através de processos de negociação entre todas as partes interessadas.

\section{CONCLUSÕES}

A localização e concentração na área central do Porto de atividades e organizações diversas reflete a presença de um amplo leque de competências instaladas que podem potenciar processos de fertilização cruzada, propiciando a prosperidade do ecossistema de empreendedorismo e inovação da área, com potencial para dar resposta efetiva aos objetivos definidos a nível regional. Enquadrando-se nos domínios de especialização inteligente definidos para a Região Norte, destacam-se na área central do Porto os domínios do Capital Simbólico, das Tecnologias e Serviços de Turismo, do Capital Humano e Serviços Especializados, da Cultura, Criação e Moda e dos Recursos do Mar e Economia. São diversas as atividades económicas presentes na área central do Porto que se enquadram nestes domínios de especialização: os serviços mais especializados, com forte potencial ao nível do capital humano; as atividades da economia cultural e criativa ou o comércio a retalho, restauração e demais atividades que servem o setor do turismo são exemplos de atividades que vão ao encontro da estratégia de RIS3. Estratégia esta também vertida na atuação do Município. Ao nível da estratégia pública, verifica-se a aposta em setores basilares e efervescentes na atualidade, com potencialidades para alavancar processos de empreendedorismo, de crescimento económico e de reforço da competitividade da área em análise, assim como da cidade no seu todo. Os desafios que se colocam prendem-se com a resiliência de atividades e do próprio território e com a capacidade dos diversos agentes atuarem em rede, concertando interesses e dirimindo eventuais pontos de clivagem, em prol do reforço do ecossistema de inovação local.

\section{BIBLIOGRAFIA}

CARAYANNIS, E. G., \& CAMPBELL, D. F. J. (2011). Open Innovation Diplomacy and a 21st Century Fractal Research, Education and Innovation (FREIE) Ecosystem: Building on the Quadruple and Quintuple Helix Innovation Concepts and the "Mode 3" Knowledge Production System. Journal Of The Knowledge Economy, 2(3), 327-372. doi:10.1007/s13132-011-0058-3.

CARVALHO, L. (2013). Knowledge Locations in Cities: Emergence and Development Dynamics (No. EPS-2013-274-S\&E). ERIM Ph.D. Series Research in Management. Erasmus Research Institute of Management. Retrieved from http://hdl.handle.net/1765/38449.

CCDR-N. (Sem data). Norte 2020: Estratégia Regional de Especialização Inteligente. Comissão de Coordenação e Desenvolvimento Regional do Norte Retrieved from https://www.portugal2020.pt/Portal2020/Media/Default/Docs/EstrategiasElnteligente/ EREI\%20Norte.pdf. 
CE. (03/03/2010). EUROPA 2020: Estratégia para um crescimento inteligente, sustentável e inclusivo. Bruxelas: Comissão Europeia Retrieved from http://eur-lex.europa.eu/LexUriServ/LexUriServ.do?uri=COM:2010:2020:FIN:pt:PDF.

ESPON. (2013). ESPON Atlas: Territorial Dimensions of the Europe 2020 Strategy E. Union (Ed.) (pp. 68). Retrieved from http:// www.espon.eu/main/Menu_Publications/Menu_ATLAS2020/.

EU. (2012). Delivering on the Europe 2020 Strategy: Handbook for Local and Regional Authorities. European Union Retrieved from https://portal.cor.europa.eu/europe2020/SiteCollectionDocuments/Europe\%202020\%20Handbook\%20for\%20Local\% 20and\%20Regional\%20Authorities.pdf.

EU, \& UN-Habitat. (2016). The State of European Cities 2016. Retrieved from http://ec.europa.eu/regional_policy/en/policy/ themes/urban-development/cities-report.

FERREIRA, C. (2013). Redes de inovação e políticas públicas: conceitos, modelos analíticos, abordagens empíricas e preocupações das políticas na atualidade. Revista de Geografia e Ordenamento do Território (GOT)(4 (dezembro)), 109-128.

FERREIRA, C. M. F., MARQUES, T. S., \& GUERRA, P. (2016a). Bottom-up Initiatives of Economic and Symbolic Innovation in Oporto City Centre. In T. d. Noronha \& H. Pinto (Eds.), Innovation for Resilience (pp. 149-168): Universidade do Algarve. Retrieved from http://www.cieo.pt/downloads/DIGITAL_Innovation_for_Resilience.pdf.

FERREIRA, C., MARQUES, T., \& GUERRA, P. (2016b). Tourism Dynamics and Architectural, Cultural and Symbolic Heritage: The Case of Oporto City Centre. In C. H. N. Henriques, M. C. Moreira, \& P. A. B. César (Eds.), Tourism and History: World Heritage - Case Studies of Ibero-American Space (Vol. III, pp. 53-72). Braga: CICS.NOVA.UMinho. Retrieved from http:// www.lasics.uminho.pt/ojs/index.php/cics_ebooks/issue/view/212.

MARQUES, T. S., QUEIROZ, J. P., \& ALVES, P. (2014). AMP 2020 Estratégia de Base Territorial. Área Metropolitana do Porto. Retrieved from http://portal.amp.pt/media/documents/2015/06/11/estrategia_amp_2020.pdf.

MARQUES, T. S., SANTOS, H. (2013), Lugares e redes de inovação na área metropolitana do Porto, Geografia: Revista da Faculdade de Letras da Universidade do Porto, Vol. 2, p.203 - 225.

MARQUES, T. S., QUEIRÓS, J. P., MENDONÇA, J. M., SANTOS, H., GUERRA, P., BABO, A. P., MACHADO, R. V., CARVALHO, L. (2017). AMP 2020: Crescimento Inteligente. Porto: Caleidoscópio - Edição e Artes Gráficas, SA, Área Metropolitana do Porto.

NIJKAMP, P., \& KOURTIT, K. (2013). The "New Urban Europe": Global Challenges and Local Responses in the Urban Century. European Planning Studies, 21(3), 291-315. doi:10.1080/09654313.2012.716243.

OECD. (2013). Innovation-driven Growth in Regions: The Role of Smart Specialisation - Preliminary Version. Retrieved from http:// www.oecd.org/sti/inno/smart-specialisation.pdf.

PORTO CITY HALL. (2016). Porto Start \& Scale Guide. 77.

RIBEIRO, J. M. F., \& FERRÃO, J. (2014). Noroeste Global. Lisboa: Fundação Calouste Gulbenkian.

SANTOS, H., MARQUES, T. S. (2013), Lugares e redes de conhecimento na área metropolitana do Porto, Geografia: Revista da Faculdade de Letras da Universidade do Porto, Vol. 2, p.179 - 202.

WINDEN, W. V., \& CARVALHO, L. (2015). Triple Helix (3H): Where are Europe's cities standing? In URBACT (Ed.), New Urban Economies: How can cities foster economic development and develop 'new urban economies' (pp. 10-14). Saint-Denis, France: URBACT. 